\title{
Agreement between retrospectively assessed health-related quality of life collected 1 week and 12 months post- injury: an observational follow-up study
}

\author{
Juanita Haagsma ${ }^{1 *}$, Gouke Bonsel $^{1}$, Mariska de Jongh ${ }^{2}$ and Suzanne Polinder ${ }^{1}$
}

\begin{abstract}
Background: Retrospective assessment of pre-injury health-related quality of life (HRQL) is frequently used to measure change from pre- to post-injury HRQL. However, retrospective measurement may be confounded by recall bias. It is assumed that presence of recall bias is influenced by several factors, such as the measurement scale or the instrument that is used, the measurement schedule, and the presence of a substantial health event during the follow up period. This study empirically tests these assumptions by comparing pre-injury EQ-5D summary scores, EQ-5D profiles and visual analogue scale (EQ-VAS) scores of trauma patients, as recorded 1 week and 12 months post-injury, respectively.
\end{abstract}

Methods: A sample of 5371 adult trauma patients who attended the Emergency Department (ED) followed by hospital admission, received postal questionnaires 1 week (T1) and 12 months (T2) post-injury. The questionnaires contained items on pre-injury health, in terms of EQ-5D3L and EQ-VAS.

Results: One thousand one hundred sixty-six completed data pairs with $\mathrm{T} 1$ and T2 pre-injury data were available. Mean pre-injury EQ-5D summary scores were 0.906 (T1) and 0.905 (T2), respectively, with moderate intertemporal agreement (intraclass correlation coefficient $($ ICC) T1T2 $=0.595)$. In absolute terms, $442(37.9 \%)$ respondents reported a different pre-injury EQ-5D profile at T2 compared to T1. The least stable EQ-5D dimension was pain/ discomfort (20.2\% reported a change). Mean T2 pre-injury EQ-VAS score was significantly higher than mean T1 pre-injury EQ-VAS score (T2 84.6 versus T1 83.3). Multivariable logistic regression analysis indicated that lower educational level, comorbid disease and having PTSD symptoms were independent predictors of change of pre-injury EQ-5D profile.

Conclusions: Despite one third of respondents reported a different pre-injury health level, if asked for on two interview occasions separated by 1 year, on the group level this difference was nil (EQ-5D summary score) to small (EQVAS). The consistent symmetrical pattern of change suggests random error to play the largest role. Intertemporal reliability was the same in EQ-5D profiles vs. EQ-VAS scores, ruling out scale effects. Particularly certain trauma subgroups showed highest distortion. While group comparisons may be trusted, in pre-post analysis and repeated measure analysis the individual injury impact and recovery pattern may be wrongly estimated.

Keywords: Health-related quality of life, EQ-5D, Recall bias, Trauma population

\footnotetext{
* Correspondence: j.haagsma@erasmusmc.nl

'Department of Public Health, Erasmus MC, University Medical Center

Rotterdam, P.O. Box 2040, 3000, CA, Rotterdam, The Netherlands

Full list of author information is available at the end of the article
}

(c) The Author(s). 2019 Open Access This article is distributed under the terms of the Creative Commons Attribution 4.0 International License (http://creativecommons.org/licenses/by/4.0/), which permits unrestricted use, distribution, and reproduction in any medium, provided you give appropriate credit to the original author(s) and the source, provide a link to the Creative Commons license, and indicate if changes were made. The Creative Commons Public Domain Dedication waiver (http://creativecommons.org/publicdomain/zero/1.0/) applies to the data made available in this article, unless otherwise stated. 


\section{Background}

Health-related quality of life (HRQL) is a prominent outcome measure in trauma care. HRQL measurement is widely applied in clinical studies, in public health as an estimator of injury impact, as source for patient information, and as indicator (patient reported outcome measurement, PROM) to compare trauma unit performance. For the last purpose, measuring individual change (e.g. from pre- to post-injury HRQL) is critical.

So far two studies obtained prospectively measured pre-injury HRQL and post-injury HRQL, though not intended for performance analysis: the Medical Expenditure Panel Survey (MEPS) [1] and the Seguimiento Universidad de Navarra (SUN) [2] cohort study. However, the impracticability of prospectively collecting HRQL data of trauma patients due to the mere unpredictability of injury events forces clinicians and researchers to rely on retrospective measurement of pre-injury heath as second best. In published studies the period at which pre-injury HRQL is measured ('retrospective window') ranges from immediately at admission [3] to 2 years post-injury $[4,5]$. Although retrospective assessment of pre-injury HRQL is much easier to implement than a pre-measurement strictu sensu, the measurement is subject to random error and recall bias $[6,7]$.

We define recall bias as a systematic measurement error, due to selective memory or other content-related reporting effects. The usual direction of recall bias is that - from a current healthy standpoint - poor health in the past is memorized as even more deteriorated as it actually was. The reverse may also happen: from an ill-health standpoint, past health may be memorized better than it actually was. We must distinguish these reporting biases from response shift, which is not a measurement error, but a true change of currently measured outcome due to a (permanent) change of the measurement perspective of the respondent ('internal measurement scale'), where the usual change is towards adaptation and reduction of cognitive dissonance if present. As a natural consequence patients may both upgrade current impaired health, or downgrade past health. Recall bias causes larger contrasts between healthy pre-state and affected post-state as indicated, while response shift - primarily relevant when health deteriorates - reduces the past-present contrast. Whatever reporting effect may be present, measured pre-post changes may not reflect the true change in HRQL.

Recall bias depends on several factors, some of which are a researcher's choice. Firstly, recall bias depends on the scale or instrument that is used. Subjective scales, like a visual analogue scale (VAS), may be more easily distort response than a classification or category scale, such as the EQ-5D5L, the Health Utilities Index (HUI), and the Short Form (SF)-36 [8]. This is particularly true if the question phrasing focuses on internal experience or internal values, rather than referring to tangible, observable, labels. Secondly, the measurement schedule matters: the longer the time interval between measurement and situation to be judged, the larger the bias, also because of the increasing risk of a substantial health event, such as a subsequent injury, a medical intervention or deterioration of a pre-existing chronic illness, that may occur between the assessments [6].

Knowledge on recall bias is vital to routine evaluation of injury care. This study aimed to analyze the presence of recall bias, if any, by comparing pre-injury EQ-5D summary scores, EQ-5D profiles and EQ-VAS scores collected shortly after and 12 months after injury.

\section{Methods}

\section{Study design}

This study is linked to the Brabant Injury Outcome Surveillance (BIOS) study. The BIOS study is a prospective observational 12-month follow-up study of trauma patients that has been admitted in one of the 11 hospitals of the Dutch Network Emergency Care Brabant [9]. The BIOS study was approved by the Medical Ethics Committee Brabant, the Netherlands (NL50258.028.14). The BIOS study protocol that was approved included the reported measures and analysis that are presented here.

\section{Participants}

Patients were eligible for inclusion if they met the following inclusion criteria: patients were aged 18 years or older and were seen at the Emergency Department (ED) of one of the 11 hospitals of Network Emergency Care Brabant and were admitted to a ward or an Intensive Care unit (ICU) and survived to hospital discharge. The sample of eligible patients consisted of victims of intentional and unintentional injury, the sustained injuries varied from moderate to severe injury, single and multiple injury. Patients who had a proven pathological ('spontaneous') fracture, insufficient knowledge of the Dutch language, or who had no permanent address of residence were excluded. Patients were recruited between March 2016 and November 2016 and were invited to participate at 1 week post-trauma.

Each injury patient that met the inclusion criteria of the study received a postal questionnaire 1 week (T1) and 12 months (T2) after the initial treatment of the injury. It was confirmed that the patient was not deceased before the questionnaire was sent. For these questionnaires the patients needed to give permission by an informed consent form. Informed consent forms were sent together with the $\mathrm{T} 1$ questionnaire. A reminder was sent to $\mathrm{T} 1$ and $\mathrm{T} 2$ non-responders, aiming to increase response rates. A participant number was assigned to the eligible patients that met the inclusion criteria of the study and this number was used to link the $\mathrm{T} 1$ and $\mathrm{T} 2$ responses. 


\section{Questionnaires}

The T1 and T2 questionnaires were strongly related, and included items regarding socio-demographics (age, gender and educational level). Additionally, T1 questionnaire included 21 items regarding the presence of one or more chronic disease(s) prior to the injury to assess comorbidity [10], e.g. chronic non-specific lung disease, heart disease, diabetes, backache, arthrosis, rheumatoid arthritis. Comorbidity was defined as the presence of any co-existing medical condition or disease process additional to the injury that qualified for inclusion [11].

The questionnaires also contained a HRQL module, consisting of the EQ-5D3L classification and the EQ-VAS. The EQ-5D3L classification invites the respondent to assign him/herself to one of three ordinated function levels (grades), in five separate dimensions: mobility, self-care, usual activities, pain/discomfort and anxiety/depression $[12,13]$. The T1 and T2 questionnaires included modules for current and for pre-injury EQ-5D3L. By combining the answers of the EQ-5D3L a numerical summary score can be derived called a utility weight. The summary score is computed by a formula that firstly yields a partial weight score for each dimension separately, depending on the reported level for that dimension, and secondly adds these partial utility weights to a score between 0 and 1 commonly. The set of weights per level and per dimension ('tariff') has been derived at an earlier stage from preference data of the population [14]. Here the Dutch tariff was used [15]. Apart from the classification, the HRQOL-modules contained the EQ-5D VAS. The EQ-VAS score ranges from 0 to 100, indicating the "the worst health you can imagine" to "the best health you can imagine".

\section{Injury data}

For our study cohort, injury related characteristics, including the Abbreviated Injury Scale (AIS) [16] and the Injury Severity Score (ISS) [17], were already available from the Brabant Trauma Registry. The ISS is a scoring system that provides an overall score for patients with multiple injuries that ranges from 0 (no injury) to 75 . The ISS is calculated by first determining the highest AIS severity code in each of the three most severely injured ISS body regions (head or neck including cervical spine, face, chest, abdomen or pelvic contents, extremities or pelvic girdle and external) and subsequently squaring and summing the numbers of these AIS severity codes. Generally, an ISS score $\geq 16$ is considered major trauma [18].

\section{Posttraumatic stress symptoms}

Posttraumatic stress disorder (PTSD) is highly prevalent in trauma populations and according to the Diagnostic and Statistical Manual of Mental Disorders (DSM) IV memory disturbances are part of the diagnostic criteria of PTSD [19]. Therefore, in our study PTSD symptoms were included as a determinant of memory problems. In our study the impact of event scale (IES) was used to assess symptoms of posttraumatic stress indicative of PTSD 12 months post-injury [20]. The IES consists of 15 items, which measure intrusive re-experiences of the trauma and avoidance of trauma-related stimuli. From the responses on the 15 items the total IES-score, ranging from 0 through 75, can be calculated. Wohlfarth et al. showed that, if 35 points are chosen as cut-off score, and if the multi-criteria PTSD diagnosis of the DSM-IV was chosen as verified outcome, that the sensitivity was 0.89 , and the specificity was 0.94 [21]. We adhered to this 35 cut off point. The Dutch translation of the IES has been found to be valid and reliable [22].

\section{Hypotheses}

We formulated four hypotheses:

- Recalled pre-injury HRQL - assumed to be a healthy state, which cannot change ex post - is reported worse with increasing retrospective window, due to recall bias.

- The gap between T1 and T2 pre-injury HRQL is larger among severely injured patients (ISS $\geq 16$ ): the cognitive dissonance effect is stronger because particularly in this group of patients rehabilitation is long and patients adapt to their non-optimal post-state.

- The gap between T1 and T2 pre-injury HRQL is larger among patients with PTSD, because this group is presumed to be affected by memory disturbances due to PTSD.

- Agreement between T1 and T2 pre-injury EQ-VAS is lower compared to T1 and T2 agreement in EQ5D3L terms, as EQ-VAS is more subjective and thus more prone to recall bias.

The first hypothesis is related to the effect of the measurement schedule on recall bias, whereas the second and third hypotheses are related to effect of high impact events and their direct or indirect effect on recall bias. The last hypothesis is related to the effect of the measurement scale on recall bias.

\section{Statistical analysis}

For analysis of the data the Statistical Package for the Social Sciences version 23 was used (SPSS Inc., Chicago, Ill). Chi-square statistics (dichotomous variables) and Fisher's exact tests were used to test for differences between the non-respondents and the respondents. Non-parametric variables (age) were tested using the Mann-Whitney U-test. 
For the analysis only complete pairs of T1-T2 responses were selected. Independent $\mathrm{t}$-tests were used to compare subgroups within the T1 responses (EQ-5D summary scores, EQ-VAS scores): males vs. females, those aged $<65$ vs. $\geq 65$ years, absent vs. present pre-existing comorbidity, ISS $<16$ vs. ISS $\geq 16$, and absence vs. presence of PTSD. One-way analysis of variance (ANOVA) was used to compare EQ-5D-summary and EQ-VAS scores on T1, according to educational attainment (three levels). We compared T1 with T2 group wise, to test the hypotheses on the size of the retrospective window, the injury severity and PTSD, with paired t-tests. We compared T1-T2 EQ-5D summary scores and EQ-VAS scores for all respondents and for subgroups (gender, age, educational level, comorbidity status, injury severity level and PTSD status).

With the intraclass correlation coefficient (ICC) we tested the similarity of the $\mathrm{T} 1$ and $\mathrm{T} 2$ scores for pre-injury EQ-5D summary score and EQ-VAS score. The ICC both catches intercept and constant effects between T1 and T2. We constructed Bland-Altman plots with limits of agreement to visually represent agreement between T1 and T1 EQ-5D and EQ-VAS. We also investigated differences between T1 and T2 pre-injury EQ-5D profiles with some detail, by dichotomizing the T1-T2 change: any level change was assigned score 1 , otherwise it was 0 . We finally used the five level indicators of the EQ-5D as numerical variable, resulting in a simple profile sum. The change of this sum was used to compare $\mathrm{T} 2$ with T1, which assumes that an upward level change in one domain may compensate a downward level change on another domain (levels $1 / 0 /-1$ ).

We predicted a change, either increase or decrease, in pre-injury EQ-5D profile and EQ-VAS (yes or no) from the socio-demographic factors, injury severity level (ISS $<16$ and ISS $\geq 16$ ) and PTSD status (yes or no). Univariate and multivariable logistic regression analysis was applied, with backward deletion (deselection criterion $p<0.05$ ).

Overall $P$-values $<0.05$ were considered to indicate statistical significance.

\section{Results}

\section{Study population}

The response rate of the first follow-up survey (T1) was $26.5 \%(n=1518)$, of which $759(50.0 \%)$ completed the survey within two weeks of sustaining the injury. The response rate of the 12-month follow-up survey (T2) was $82.7 \%(n=1255)$. The T1 and T2 pre-injury EQ-5D was completed by 1166 persons. Respondents were significantly younger than non-respondents (median age 62 versus median age $66, p<0.05$ ), the proportion males was higher (non-responders: $53 \%$ male versus responders: $46 \%$ males, $p<0.05$ ).

\section{EQ-5D summary scores}

Group wise comparisons - Mean EQ-5D summary scores at $\mathrm{T} 1$ and $\mathrm{T} 2$ were 0.906 and 0.905 respectively (see Table 1). Lower pre-injury EQ-5D summary score was associated with being female, being older, lower educational level and the presence of pre-existing comorbidity (see Table 1). There was no difference in mean T1 and T2 pre-injury EQ-5D summary scores, except for patients without pre-existing morbidity. This group of patients had significantly lower recalled pre-injury EQ-5D scores at T2, compared to T1 $(p<0.05)$.

Pairwise comparisons - Pre-injury EQ-5D summary scores measured at T1 and T2 were moderately similar $(\mathrm{ICC}=0.595)$. This was consistent with the Bland-Altman plot in Fig. 1. Figure 1 shows the differences between the two pre-injury EQ-5D summary scores plotted against the averages of the two pre-injury EQ-5D summary scores. For example, if a patient has T1 and T2 pre-injury EQ-5D summary scores of 0.89 and 0.81 , respectively, than the mean T1-T2 pre-injury utility scores is 0.85 and the difference in T1-T2 pre-injury utility scores is 0.08 . The Bland-Altman plot in Fig. 1 covers a moderate proportion of the possible change scores. The difference between T1 and T2 utility scores is largest when an average T1-T2 utility score is between 0.4 and 0.8 .

The inter-temporal agreement between the T1 and T2 scores was lowest in the subgroup no pre-existing morbidity ( $\mathrm{ICC}=0.408$ ) and highest in the subgroup $\geq 65$ years $(\mathrm{ICC}=0.651)$ and patients with unknown ISS $(\mathrm{ICC}=$ $0.825)$.

\section{Pre-injury EQ-5D profile}

Fifty two out of $243(21.4 \%)$ possible EQ-5D profiles were reported at T1 and T2. An optimal health profile 11111 was reported by 727 patients $(62.3 \%)$ at T1 and 757 patients $(64.9 \%)$ at $\mathrm{T} 2$.

In total, 724 (62.1\%) of 1166 respondents had identical EQ-5D profiles at T1 and T2 (see Table 2). Of these, the majority $(n=634 ; 87.6 \%)$ had the optimal health profile 11111. Of the changed respondents, only 93 (21.0\%) had a T1 profile of 11111 . Half of the changed respondents reported more, the other half reported less problems at T2, indicating random error. Twenty seven respondents (2.3\%) reported a different pre-injury EQ-5D profile at $\mathrm{T} 1$ and $\mathrm{T} 2$, but still had a similar $\mathrm{T} 1$ and $\mathrm{T} 2$ summary score. The EQ-5D dimensions that differed most frequently between $\mathrm{T} 1$ and $\mathrm{T} 2$ were pain/discomfort (20.2\% of the respondents with a change), mobility (13.1\%) and daily activities (13.0\%).

\section{Pre-injury EQ-VAS score}

Group wise comparisons - Mean EQ-VAS scores at T1 and T2 were 82.9 and 84.4, respectively (see Table 3). Lower pre-injury EQ-VAS scores were associated with 
Table 1 Mean pre-injury EQ-5D summary score assessed 1 week (T1) and 12 months (T2) post-injury and intraclass correlation coefficients (ICC)

\begin{tabular}{|c|c|c|c|c|c|}
\hline & $\mathrm{n}$ & $\begin{array}{l}\text { Pre-injury EQ-5D assessed } \\
\text { at T1 (1 week post-injury) }\end{array}$ & $\begin{array}{l}\text { Pre-injury EQ-5D assessed at T2 } \\
\text { (12 months post-injury) }\end{array}$ & $P$-value & ICC (95\% Cl) \\
\hline Total & $1166^{\$}$ & 0.906 (SD 0.17) & 0.905 (SD 0.17) & 0.886 & $0.595(0.56,0.63)$ \\
\hline \multicolumn{6}{|l|}{ Gender } \\
\hline Males & 615 & 0.930 (SD 0.14) & 0.922 (SD 0.16) & 0.208 & $0.540(0.48,0.59)$ \\
\hline Females & 551 & 0.879 (SD 0.18) & 0.886 (SD 0.18) & 0.324 & $0.626(0.57,0.67)$ \\
\hline \multicolumn{6}{|l|}{ Age } \\
\hline$<65$ years & 575 & 0.940 (SD 0.12) & 0.932 (SD 0.16) & 0.157 & $0.525(0.46,0.58)$ \\
\hline$\geq 65$ years & 591 & 0.872 (SD 0.19) & 0.879 (SD 0.18) & 0.343 & $0.651(0.56,0.66)$ \\
\hline \multicolumn{6}{|l|}{ Educational level } \\
\hline Low & 309 & 0.849 (SD 0.22) & 0.862 (SD 0.20) & 0.242 & $0.551(0.47,0.62)$ \\
\hline Middle & 450 & 0.916 (SD 0.15) & 0.914 (SD 0.17) & 0.760 & $0.640(0.58,0.69)$ \\
\hline High & 380 & 0.943 (SD 0.11) & 0.936 (SD 0.14) & 0.217 & $0.539(0.46,0.61)$ \\
\hline Unknown & 27 & 0.851 (SD 0.15) & 0.809 (SD 0.20) & 0.214 & $0.535(0.21,0.76)$ \\
\hline \multicolumn{6}{|l|}{ Comorbidity status } \\
\hline No comorbidity & 498 & 0.975 (SD 0.08) & 0.960 (SD 0.11) & $0.003^{*}$ & $0.408(0.33,0.48)$ \\
\hline Comorbidity & 649 & 0.851 (SD 0.19) & 0.862 (SD 0.20) & 0.138 & $0.575(0.52,0.63)$ \\
\hline Unknown & 19 & 0.955 (SD 0.09) & 0.919 (SD 0.13) & 0.152 & $0.505(0.10,0.77)$ \\
\hline \multicolumn{6}{|l|}{ Injury Severity Score } \\
\hline ISS $<16$ & 1093 & 0.905 (SD 0.17) & 0.904 (SD 0.17) & 0.907 & $0.590(0.55,0.63)$ \\
\hline ISS $>=16$ & 65 & 0.928 (SD 0.16) & 0.924 (SD 0.19) & 0.813 & $0.636(0.47,0.76)$ \\
\hline Unknown & 8 & 0.819 (SD 0.13) & 0.820 (SD 0.18) & 0.980 & $0.825(0.35,0.96)$ \\
\hline \multicolumn{6}{|l|}{ PTSD status ${ }^{\&}$} \\
\hline No PTSD (IES < 35) & 696 & 0.927 (SD 0.14) & 0.932 (SD 0.13) & 0.249 & $0.564(0.51,0.61)$ \\
\hline PTSD (IES > =35) & 121 & 0.829 (SD 0.23) & 0.816 (SD 0.25) & 0.514 & $0.582(0.45,0.69)$ \\
\hline Unknown & 349 & 0.891 (SD 0.18) & 0.882 (SD 0.20) & 0.325 & $0.592(0.52,0.66)$ \\
\hline
\end{tabular}

$S D$ standard deviation, ICC Intraclass correlation coefficient, ISS injury severity score

$\$$ Patients who completed the pre-injury EQ-5D shortly after and 12 months after sustaining an injury \& PTSD status was measured with the Impact of Event Scale (IES) 12 months post-injury ${ }^{*} p<0.05$

being female, being older, lower educational level, the presence of pre-existing comorbidity and having PTSD symptoms indicative of PTSD (see Table 3). The T2 pre-injury EQ-VAS scores were significantly higher than the T1 pre-injury VAS scores. This was true for each sociodemographic subgroup of patients, but the difference was only significant for subgroups females, patients aged 65 and older, and patients with lower and middle educational level. Patients with less injury impact (ISS < 16, PTSD absent) showed considerably larger T1-T2 gap where T1 was worse.

An EQ-VAS score of 100 was reported by 182 patients (16.1\%) at T1 and 177 patients (15.6\%) at T2. Of these patients, respectively 92 and 89 reported an EQ-5D of 11111. Approximately one in seven patients $(n=172$, $15.2 \%)$ had a difference of 10 points or more between the $\mathrm{T} 1$ and T2 pre-injury EQ-VAS score in either direction.
Pairwise comparisons - Pre-injury EQ-VAS scores at $\mathrm{T} 1$ and $\mathrm{T} 2$ corresponded moderately $(\mathrm{ICC}=0.580)$. This was visualized with the Bland-Altman plot in Fig. 2. Figure 2 shows the differences between the two pre-injury EQ-VAS scores plotted against the averages of the two pre-injury EQ-VAS scores. The Bland-Altman plot in Fig. 2 covered a moderate proportion of the possible change scores.

\section{Factors associated with change in pre-injury EQ-5D profile and EQ-VAS score}

EQ-5D profile - Univariate logistic regression analyses showed that females, older age, lower educational level, having co-morbid disease and having PTSD were significantly associated with a change, either increase or decrease, of EQ-5D profile at T1 versus T2. Multivariable logistic regression analysis indicated that having comorbid 


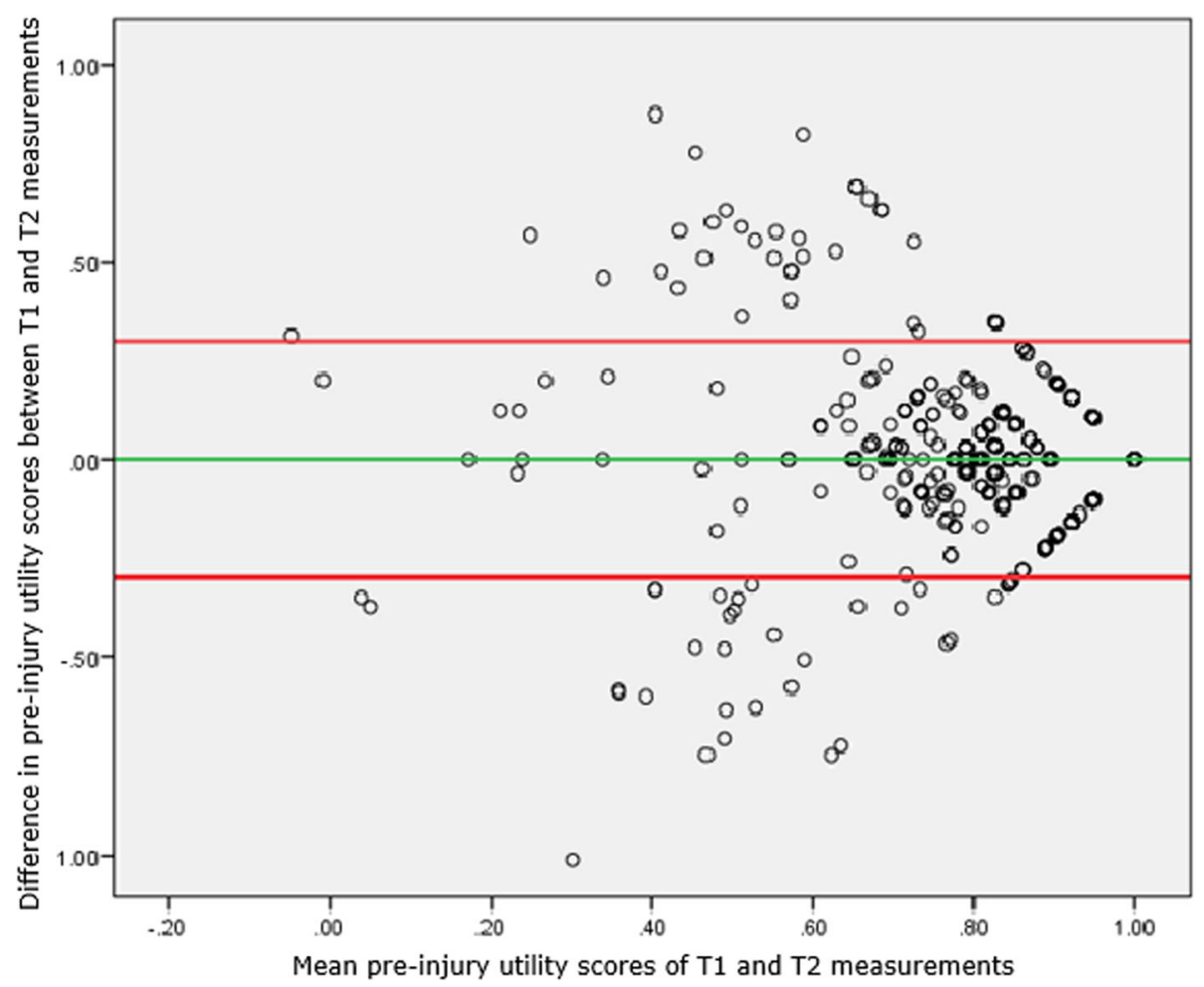

Fig. 1 Bland-Altman plot for agreement in T1 and T2 pre-injury EQ-5D utility scores

disease (odds ratio (OR) 3.3, 95\% CI 2.3-4.7), having PTSD symptoms indicative of PTSD (OR 2.3; 95\% CI, 1.5 to 3.6 ) and increasing age (OR 1.02 ; $95 \% \mathrm{CI}, 1.01$ to 1.03 ) are independent factors that are associated with a change of T2 EQ-5D profile compared to T1.

EQ-VAS - Univariate logistic regression analyses showed that none of the factors were significantly associated with a difference of $>1$ EQ-VAS point between $\mathrm{T} 1$ and $\mathrm{T} 2$.

Table 2 Correspondence of pre-injury EQ-5D profile assessed 1 week (T1) and 12 months (T2) post-injury

\begin{tabular}{lllll}
\hline Pre-injury EQ-5D & $\mathrm{n}$ & $\mathrm{T} 1=\mathrm{T} 2$ & $\mathrm{~T} 1<\mathrm{T} 2$ & $\mathrm{~T} 1>\mathrm{T} 2$ \\
\hline EQ-5D profile & 1166 & $62.1 \%^{\mathrm{a}}$ & $18.2 \%^{\mathrm{a}}$ & $18.3 \%^{\mathrm{a}}$ \\
Mobility & 1166 & $86.9 \%$ & $6.2 \%$ & $6.9 \%$ \\
Self-care & 1166 & $93.0 \%$ & $3.4 \%$ & $3.6 \%$ \\
Usual activities & 1166 & $87.0 \%$ & $6.8 \%$ & $6.2 \%$ \\
Pain/discomfort & 1166 & $79.8 \%$ & $9.5 \%$ & $10.7 \%$ \\
Anxiety/depression & 1166 & $90.1 \%$ & $6.2 \%$ & $3.8 \%$
\end{tabular}

$\mathrm{T} 1=\mathrm{T} 2$ : respondents filled in exactly the same pre-injury EQ-5D profile at $\mathrm{T} 1$ and $\mathrm{T} 2$

$\mathrm{T} 1<\mathrm{T} 2$ : respondents that reported less problems with the EQ-5D at $\mathrm{T} 2$

$\mathrm{T} 1>\mathrm{T} 2$ : respondents that reported more problems with the EQ-5D at T2

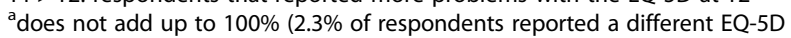
profile at $\mathrm{T} 1$ and $\mathrm{T} 2$, but the profile summary score of the profiles were the same)

\section{Discussion \\ Main findings}

Our study showed that pre-injury health status as measured with EQ-5D3L showed expected patterns according to e.g. age and morbidity. A larger retrospective window (1 year vs 1 week) generally did not systematically influence the reported HRQL levels. Pre-injury health status as measured with EQ-5D3L one week versus one year after the injury did not show any systematic difference on the group level. It showed, however, that a substantial number of respondents showed small changes of health profile or EQ-VAS, in both directions. The consistent symmetrical pattern of change suggests that changes predominantly are the result of random error, rather than bias. The factors associated with change into any direction (older age, co-morbid disease, PTSD) may be factors associated with precision of these measures as such.

The least stable EQ-5D dimension between $\mathrm{T} 1$ and T2 was pain/discomfort. This may be due to the fact that pain/discomfort is a more subjective dimension compared to mobility, self-care and usual activities [23]. However, anxiety/depression, which is also a more subjective dimension of the EQ-5D, was much more stable between $\mathrm{T} 1$ and $\mathrm{T} 2$, whereas mobility and usual activities also differed frequently between $\mathrm{T} 1$ and $\mathrm{T} 2$. 
Table 3 Mean pre-injury VAS scores assessed 1 week (T1) and 12 months (T2) post-injury and intraclass correlation coefficients (ICC)

\begin{tabular}{|c|c|c|c|c|c|}
\hline & $\mathrm{n}$ & $\begin{array}{l}\text { Pre-injury EQ-VAS assessed at T1 } \\
\text { (1 week post-injury) }\end{array}$ & $\begin{array}{l}\text { Pre-injury EQ-VAS assessed at T2 } \\
\text { (12 months post-injury) }\end{array}$ & $P$-value & ICC \\
\hline Total & 1132 & 82.9 (SD 15) & 84.4 (SD 14) & $0.001^{*}$ & $0.580(0.54,0.62)$ \\
\hline \multicolumn{6}{|l|}{ Gender } \\
\hline Males & 596 & 85.1 (SD 14) & 85.8 (SD 13) & 0.191 & $0.580(0.53,0.63)$ \\
\hline Females & 536 & 81.3 (SD 16) & 83.3 (SD 15) & $0.001^{*}$ & $0.571(0.51,0.63)$ \\
\hline \multicolumn{6}{|l|}{ Age } \\
\hline$<65$ years & 564 & 86.4 (SD 13) & 86.9 (SD 14) & 0.324 & $0.564(0.51,0.62)$ \\
\hline $65+$ years & 568 & 80.3 (SD 16) & 82.4 (SD 15) & $0.000^{*}$ & $0.565(0.51,0.62)$ \\
\hline \multicolumn{6}{|l|}{ Educational level } \\
\hline Low & 295 & 79.1 (SD 16) & 81.4 (SD 15) & $0.007^{*}$ & $0.548(0.47,0.62)$ \\
\hline Middle & 439 & 84.2 (SD 16) & 85.7 (SD 14) & $0.021^{*}$ & $0.603(0.54,0.66)$ \\
\hline High & 373 & 86.3 (SD 12) & 86.6 (SD 12) & 0.582 & $0.585(0.52,0.65)$ \\
\hline Unknown & 25 & 74.0 (SD 16) & 75.2 (SD 20) & 0.798 & $0.142(-0.24,0.48)$ \\
\hline \multicolumn{6}{|l|}{ Comorbidity status } \\
\hline No comorbidity & 486 & 89.7 (SD 12) & 90.0 (SD 10) & 0.168 & $0.423(0.35,0.49)$ \\
\hline Comorbidity & 628 & 78.4 (SD 15) & 80.4 (SD 16) & 0.083 & $0.557(0.50,061)$ \\
\hline Unknown & 18 & 84.9 (SD 11) & 87.4 (SD 12) & 0.111 & $0.353(-0.09,0.69)$ \\
\hline \multicolumn{6}{|l|}{ Injury Severity Score } \\
\hline ISS $<16$ & 1060 & 83.2 (SD 15) & 84.6 (SD 14) & $0.001^{*}$ & $0.582(0.54,0.62)$ \\
\hline ISS $>=16$ & 64 & 87.4 (SD 13) & 87.4 (SD 14) & 0.991 & $0.659(0.50,0.78)$ \\
\hline Unknown & 8 & 69.4 (SD 23) & 69.6 (SD 27) & 0.979 & $0.448(-0.31,0.86)$ \\
\hline \multicolumn{6}{|l|}{ PTSD symptoms ${ }^{\&}$} \\
\hline No PTSD $($ IES < 35) & 629 & 84.9 (SD 13) & 86.1 (SD 12) & $0.003^{*}$ & $0.655(0.61-0.70)$ \\
\hline PTSD (IES > =35) & 111 & 78.4 (SD 17) & 79.0 (SD 19) & 0.869 & $0.495(0.35,0.62)$ \\
\hline Unknown & 320 & 81.5 (SD 17) & 83.5 (SD 15) & $0.022^{*}$ & $0.515(0.43,0.59)$ \\
\hline
\end{tabular}

SD standard deviation, ICC Intraclass correlation coefficient, ISS injury severity score

\&PTSD symptoms measured with the Impact of Event Scale (IES) 12 months post-injury ${ }^{*} p<0.05$

The EQ-5D summary scores and EQ-VAS scores of respondents with severe (ISS $>16$ ) injuries and respondents with PTSD did not differ significantly. Kwong et al. argued that, compared to continuous measures, the more restricted range of responses of generic measures, such as the EQ-5D-3 L, may lead to smaller variability in scores [24]. In our study this may have led to greater homogeneity in pre-injury EQ-5D assessed at T1 and T2 compared to pre-injury EQ-VAS. However, our findings also show that pre-injury EQ-5D was just as easily distorted as the EQ-VAS. More than one in three respondents reported a different pre-injury EQ-5D health state at $\mathrm{T} 1$ compared to $\mathrm{T} 2$, whereas only one in eight reported a different pre-injury EQ-VAS score at T1 compared to T2.

\section{Comparison to previous studies}

Many studies have retrospectively collected pre-injury HRQL [25]. However, to our knowledge none of these studies have assessed pre-injury HRQL at multiple points in time post-injury. Litwin and McGuigan (1999) did compare retrospectively collected health status data at multiple follow up assessments. In their study Litwin and McGuigan investigated agreement of pre-injury HRQL in men who had undergone radical prostatectomy for early-stage prostate cancer using the RAND 12-Item Short-Form Health Survey (SF-12) [26]. No differences between recollected pre-event SF-12 HRQL between 7 and 37 months port-surgery were found. Howell et al. (2008) who investigated retrospectively collected health status after total hip replacement also did not find systematic differences between recalled pre-event SF-12 between 3 days and 3 months after surgery [27]. This is in agreement with the findings of our study, even though our study differed with regards to several factors, such as difference in time period of pre-event HRQL assessments, difference in the event, difference in instrument that was used to assess pre-event HRQL, and difference in patient groups that were investigated. Each of these factors may affect 


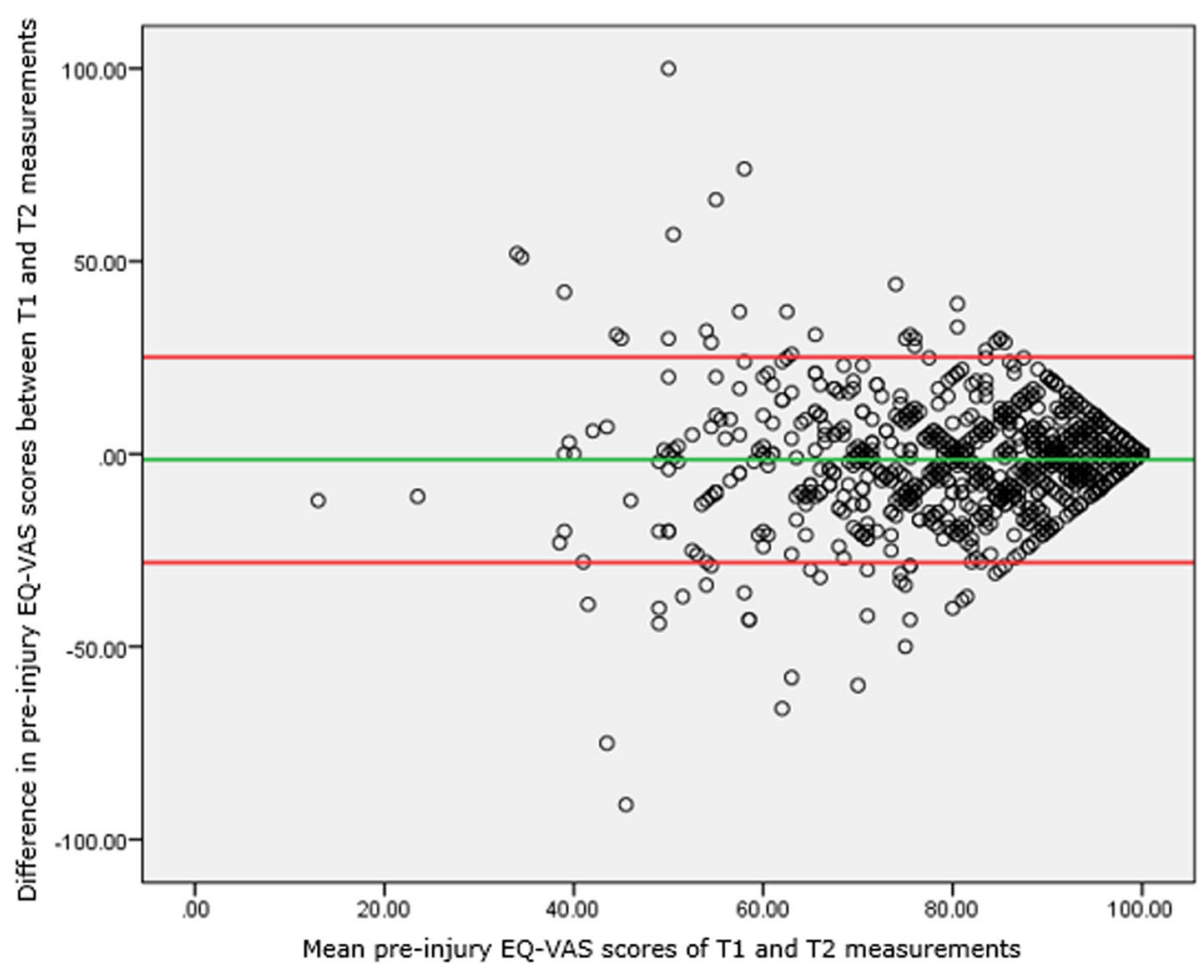

Fig. 2 Bland-Altman plot for agreement in T1 and T2 pre-injury EQ-VAS scores

agreement between retrospectively assessed pre-event HRQL measurements [8, 28].

\section{Strengths and limitations}

This study had several strengths. First, the number of respondents was high and it was therefore possible to test for differences within specific subgroups, such as patients with pre-existing comorbidity and patients who sustained severe injuries. Second, this study assessed both pre-injury EQ-VAS and pre-injury EQ-5D which allowed for comparisons of pre-injury HRQL over time on a subjective scale and a classification like scale. Third, the time period between the two follow up moments was almost one year. With this long time period between measurements it is not very likely that a respondent remembered exactly what he/she filled in at T1. A second advantage of this long time period between measurements is that it allowed us to study recall of pre-event HRQL shortly after and long after injury. The time period between the event and measurement of recalled pre-event HRQL is identified as one of the factors influencing patient recall and it is assumed that asking for a recall over longer periods leads to more recall bias [6].

A limitation of this study was the low rate of patients that responded to the surveys and that filled in the EQ-5D at both follow up moments. The low response rate in our survey may have resulted from the length of the questionnaires, which included items on pre-injury and current health as well as health care consumption and return to work. It is likely that patients with limited interest in these aspects or patients with cognitive impairments were less likely to respond. As a result, the respondents that have filled out the questionnaires may have been a biased and self-selected sample with higher consistency between two measurements and this may have affected the internal validity of our findings. Moreover, the low and possible biased sample may affect the generalizability of our results to the Dutch trauma population.

A second limitation is that we administered a stand-alone paper-and-pencil questionnaire, which did not allow us to verify if the respondents understood the HRQL questions. Our results showed that half of the respondents who reported full health on the EQ-VAS also reported one or more problems on the EQ-5D. This seems contrary to what is expected and may indicate that some of the respondents did not understand the pre-injury EQ-VAS question and/or pre-injury EQ-5D questions. Nonetheless, the findings that the mean pre-injury EQ-5D summary and EQ-VAS scores were lower for females, older respondents, respondents with pre-existing comorbidity, and patients with lower educational level corresponds to findings from other studies on HRQL [29-31]. Therefore, we assume that only a small proportion of the respondents may have had difficulty filling the pre-injury EQ-VAS question and that this had only a small effect on our findings. 


\section{Conclusions}

Despite one third of respondents reported a different pre-injury health level, if asked for on two interview occasions separated by 1 year, on the group level this difference was nil (EQ summary score) to small (EQ-VAS). The consistent symmetrical pattern of change suggests that random error plays the largest role. Intertemporal reliability was the same in EQ-5D profiles vs. EQ-VAS scores, ruling out scale effects. Particularly certain trauma subgroups showed highest distortion. While group comparisons may be trusted, in pre-post analysis and repeated measure analysis the individual injury impact and recovery pattern may be wrongly estimated.

\begin{abstract}
Abbreviations
AIS: Abbreviated Injury Scale; ANOVA: One-way analysis of variance; BIOS: Brabant Injury Outcome Surveillance; DSM-IV: Diagnostic and Statistical Manual of Mental Disorders IV; ED: Emergency Department; HRQL: Healthrelated quality of life; HUI: Health Utilities Index; ICC: Intraclass correlation coefficient; ICU: Intensive Care unit; IES: Impact of event scale; ISS: Injury Severity Score; MEPS: Medical Expenditure Panel Survey; OR: Odds ratio; PTSD: Posttraumatic stress disorder; RAND 12: Short-Form Health Survey-12. SF-36: Short Form-36; SUN: Seguimiento Universidad de Navarra; T1: Postal questionnaire 1 week after the initial treatment of the injury; T1: Postal questionnaire 12 months after the initial treatment of the injury; VAS: Visual analogue scale
\end{abstract}

\section{Acknowledgements}

We would like to thank the BIOS research team for collecting the data that were analysed in this study.

\section{Funding}

This study was funded by the EuroQoL group (Grant number EQ Project 2016160). The views expressed by the authors do not necessarily reflect the views of the EuroQol Group.

\section{Availability of data and materials}

The datasets used and/or analysed during the current study are available from the corresponding author on reasonable request.

\section{Authors' contributions}

$\mathrm{JH}, \mathrm{GB}, \mathrm{MdJ}$ and SP conceptualized the design of the study. MdJ and SP coordinated the collection of the data. JH analysed the data. $J H, G B, M d J$ and SP interpreted the data. JH and GB drafted the manuscript. JH, GB, Md and SP revised the manuscript critically for important intellectual content. GB, MdJ and SP have given final approval of the version of the manuscript that was submitted to be published. Each author agreed to be accountable for all aspects of the work in ensuring that questions related to the accuracy or integrity of any part of the work are appropriately investigated and resolved.

\section{Ethics approval and consent to participate}

This study was approved by the Medical Ethics Committee Brabant, the Netherlands. Patients needed to give permission for participation in the BIOS study. Informed consent forms were sent together with the T1 questionnaire.

\section{Consent for publication}

Not applicable

\section{Competing interests}

This study was funded by the EuroQoL group (Grant number EQ Project 2016160), of which GJB is a member.

\section{Publisher's Note}

Springer Nature remains neutral with regard to jurisdictional claims in published maps and institutional affiliations.

\section{Author details}

'Department of Public Health, Erasmus MC, University Medical Center Rotterdam, P.O. Box 2040, 3000, CA, Rotterdam, The Netherlands. ${ }^{2}$ Department Trauma TopCare, ETZ Hospital, Hilvarenbeekseweg 60, 5022, GC, Tilburg, The Netherlands.

Received: 17 August 2018 Accepted: 9 April 2019

Published online: 23 April 2019

\section{References}

1. Alghnam S, Palta M, Remington PL, Mullahy J. The association between motor vehicle injuries and health-related quality of life: a longitudinal study of a population-based sample in the United States. Qual Life Res. 2014;23(1):119-27.

2. Pons-Villanueva J, Rodriguez De Armenta MJ, Martinez-Gonzalez MA, SeguiGomez M. Longitudinal assessment of quality of life and its change in relation to motor vehicle crashes: the SUN (Seguimiento Universidad de Navarra) cohort. J Trauma Inj Infect Crit Care. 2011;70:1072-7.

3. Hagino H, Nakamura T, Fujiwara S, Oeki M, Okano T, Teshima R. Sequential change in quality of life for patients with incident clinical fractures: a prospective study. Osteoporosis Int. 2009;20:695-702.

4. Gross T, Schuepp M, Attenberger C, Pargger H, Amsler F. Outcome in polytraumatized patients with and without brain injury. Acta Anaesthesiol Scand. 2012;56:1163-74.

5. Ulvik A, Kvale R, Wentzel-Larsen T, Flaatten H. Quality of life 2-7 years after major trauma. Acta Anaesthesiol Scand. 2008;52:195-201.

6. Schmier JK, Halpern MT. Patient recall and recall bias of health state and health status. Expert Rev Pharmacoecon Outcomes Res. 2004;4:159-63.

7. Sprangers MA, Schwartz CE. Integrating response shift into health-related quality of life research: a theoretical model. Soc Sci Med. 1999;48:1507-15.

8. Stull DE, Leidy NK, Parasuraman B, Chassany O. Optimal recall periods for patient-reported outcomes: challenges and potential solutions. Curr Med Res Opin. 2009;25:929-42.

9. de Jongh MA, Kruithof N, Gosens T, van de Ree $\mathrm{CL}$, de Munter $\mathrm{L}$, Brouwers L, Polinder S, Lansink KW. Group B: prevalence, recovery patterns and predictors of quality of life and costs after non-fatal injury: the Brabant injury outcome surveillance (BIOS) study. Inj Prev. 2017;23:59.

10. CBS: Permanent onderzoek leefsituatie. . Voorburg/Heerlen: Statistics Netherlands (CBS); 2003.

11. Mosby. Mosby's medical dictionary. In: Elsevier health sciences. 8th ed; 2009.

12. Brooks R. EuroQol: the current state of play. Health Policy. 1996;37:53-72.

13. Rabin R, de Charro F. EQ-5D: a measure of health status from the EuroQol group. Ann Med. 2001;33:337-43.

14. Dolan P. Modeling valuations for EuroQol health states. Med Care. 1997;35: 1095-108.

15. Lamers LM, Stalmeier PF, McDonnell J, Krabbe PF, van Busschbach JJ. Measuring the quality of life in economic evaluations: the Dutch EQ-5D tariff. Ned Tijdschr Geneeskd. 2005;149:1574-8.

16. Gennarelli TA, Wodzin E. AIS 2005: a contemporary injury scale. Injury. 2006; 37:1083-91

17. Baker SP, O'Neill B, Haddon W Jr, Long WB. The injury severity score: a method for describing patients with multiple injuries and evaluating emergency care. J Trauma. 1974;14:187-96.

18. Boyd CR, Tolson MA, Copes WS. Evaluating trauma care: the TRISS method. Trauma Score and the Injury Severity Score. J Trauma. 1987;27:370-8.

19. Haagsma JA, Ringburg AN, van Lieshout EM, van Beeck EF, Patka P, Schipper IB, Polinder S. Prevalence rate, predictors and long-term course of probable posttraumatic stress disorder after major trauma: a prospective cohort study. BMC Psychiatry. 2012;12:236.

20. Horowitz M, Wilner N, Alvarez W. Impact of event scale: a measure of subjective stress. Psychosom Med. 1979;41:209-18.

21. Wohlfarth TD, van den Brink W, Winkel FW, ter Smitten M. Screening for posttraumatic stress disorder: an evaluation of two self-report scales among crime victims. Psychol Assess. 2003;15:101-9.

22. van der Ploeg E, Mooren TT, Kleber RJ, van der Velden PG, Brom D. Construct validation of the Dutch version of the impact of event scale. Psychol Assess. 2004;16:16-26.

23. Blome C, Augustin M. Measuring change in quality of life: bias in prospective and retrospective evaluation. Value Health. 2015;18:110-5.

24. Kwong E, Neuburger J, Black N. Agreement between retrospectively and contemporaneously collected patient-reported outcome measures (PROMs) in hip and knee replacement patients. Qual Life Res. 2018;27(7):1845-54. 
25. Scholten AC, Haagsma JA, Steyerberg EW, van Beeck EF, Polinder S. Assessment of pre-injury health-related quality of life: a systematic review. Popul Health Metrics. 2017;15:10.

26. Litwin MS, McGuigan KA. Accuracy of recall in health-related quality-of-life assessment among men treated for prostate cancer. J Clin Oncol. 1999;17: 2882-8.

27. Howell J, Xu M, Duncan CP, Masri BA, Garbuz DS. A comparison between patient recall and concurrent measurement of preoperative quality of life outcome in total hip arthroplasty. J Arthroplast. 2008;23:843-9.

28. Dorman P, Slattery J, Farrell B, Dennis M, Sandercock P. Qualitative comparison of the reliability of health status assessments with the EuroQol and SF-36 questionnaires after stroke. United Kingdom collaborators in the international stroke trial. Stroke. 1998;29:63-8.

29. Mielck A, Reitmeir P, Vogelmann M, Leidl R. Impact of educational level on health-related quality of life (HRQL): results from Germany based on the EuroQol 5D (EQ-5D). Eur J Pub Health. 2013;23:45-9.

30. Feng Y, Devlin N, Herdman M. Assessing the health of the general population in England: how do the three- and five-level versions of EQ-5D compare? Health Qual Life Outcomes. 2015;13:171.

31. Sorensen J, Davidsen M, Gudex C, Pedersen KM, Bronnum-Hansen H. Danish EQ-5D population norms. Scand J Public Health. 2009;37:467-74.

Ready to submit your research? Choose BMC and benefit from:

- fast, convenient online submission

- thorough peer review by experienced researchers in your field

- rapid publication on acceptance

- support for research data, including large and complex data types

- gold Open Access which fosters wider collaboration and increased citations

- maximum visibility for your research: over $100 \mathrm{M}$ website views per year

At BMC, research is always in progress.

Learn more biomedcentral.com/submissions 